\title{
Coarsening in an integro-differential model of phase transitions
}

\author{
Dugald B. Duncan \\ Department of Mathematics \\ Heriot-Watt University \\ Edinburgh EH14 4AS, Scotland \\ Michael Grinfeld and Iulian Stoleriu \\ Department of Mathematics \\ University of Strathclyde \\ Livingstone Tower \\ 26 Richmond Street \\ Glasgow G1 1XH, Scotland
}

Revised June 92000

\begin{abstract}
Coarsening of solutions of the integro-differential equation

$$
u_{t}=\varepsilon \int_{\Omega} J(|x-y|)(u(y)-u(x)) d y-f(u), x \in \Omega,
$$

where $\Omega \subset \mathbb{R}^{n}, J(\cdot) \geq 0, \varepsilon>0$ and $f(u)=u^{3}-u$ (or a similar bistable nonlinear term), is examined and compared with results for the Allen-Cahn partial differential equation. Both equations are used as models of solid-solid phase transitions. In particular it is shown that when $\varepsilon$ is small enough, solutions of this integro-differential equation do not coarsen, in contrast to those of the Allen-Cahn equation.

The special case $J(\cdot) \equiv 1$ is explored in detail giving insight into the behaviour in the more general case $J(\cdot) \geq 0$. Also, a numerical approximation method is outlined and used on tests in both one and two space dimensions to verify and illustrate the main result.
\end{abstract}




\section{Introduction}

The Cahn-Hilliard and the Allen-Cahn equations are well established in the applied mathematics literature as models for, respectively, order parameter preserving and order parameter non-preserving phase transition phenomena in solids. Thus, the Cahn-Hilliard equation models phase separation in binary alloys and the AllenCahn equation can be used to model transitions between variants in a crystalline substance. For a thorough introduction to the issues involved, see [6] or [13].

Recently it was suggested that the Cahn-Hilliard equation does not reproduce faithfully the details of phase separation when the forces driving the process operate at very short length scales [10]. Observations of this kind motivate the search for, and analysis of alternative models for solid-solid phase transitions. At the moment, two related directions are active. One involves considering mean field equations directly derived from the Ising model (see [14] for derivation, [5] for simulations and [10] for applications). The other, and the one we consider here was written down by van der Waals [15]; our interest is chiefly motivated by the work of Fife [7] and the discussion in Khachaturyan's book [11].

Instead of considering the Ginzburg-Landau free energy functional, we deal with gradient flows of the following free energy functional,

$$
E_{K}(u)=\frac{\varepsilon}{4} \int_{\Omega} \int_{\Omega} J(|x-y|)(u(y)-u(x))^{2} d y d x+\int_{\Omega} F(u) d x,
$$

where $\Omega$ is a domain in $\mathbb{R}^{n}$; from now on we take $|\Omega|=1$. $u(x, t)$ is the order parameter, $F(u)$ is a double-well free energy, $J(\cdot)$ is a kernel that measures interactions between particles at positions $x$ and at $y$, and $\varepsilon$ is a convenient measure of the overall strength of such interactions. The order parameter non-conserving gradient flow of this functional is the integro-differential equation (IDE)

$$
u_{t}=\varepsilon \int_{\Omega} J(|x-y|)(u(y)-u(x)) d y-f(u), x \in \Omega,
$$

where $f(u) \equiv F^{\prime}(u)$ is now a (dissipative) bistable nonlinearity. Equation (1.2) been popularized by Bates, Fife and coworkers. See [1] for a careful derivation, [7] for a discussion of its basic properties and an overview of known results, and [2] for a thorough discussion of steady state solutions.

In this paper we mainly consider coarsening (roughly speaking, the disappearance of fine structure with time) of solutions of (1.2). We follow [7] and most of the literature and only consider the case $J(\cdot) \geq 0$, but note that physical situations in which $J$ might take negative values, abound; for more on that see [2]. In addition, we take $\Omega$ to be bounded and make no particular assumptions on $J(\cdot)$ beyond taking it to be in $L^{2}(\Omega)$. For definiteness we take $f(u)=u^{3}-u$; however, the arguments below are easily adapted to deal with any bistable nonlinearity. 
We give a partial answer to Fife's question [7], which asks how far the similarities between (1.2) and the Allen-Cahn equation

$$
u_{t}=\varepsilon \Delta u-f(u), x \in \Omega, \frac{\partial u}{\partial n}=0 \text { on } \partial \Omega
$$

can be taken. In one space dimension, the situation for the Allen-Cahn equation is well understood; see the fundamental papers of Carr and Pego and of Fusco and Hale $[4,8]$. For small enough $\varepsilon$ solutions of (1.3) evolve as follows: domains where $u \approx \pm 1$ quickly appear; these are separated by sharp transition layers. A transition layer moves exponentially slowly, on an $O\left(e^{c / \sqrt{\varepsilon}}\right)$ time scale, and is annihilated by collision either with another layer or with the boundary. With probability one, solutions coarsen to one of the two stable constant solutions, $u \equiv \pm 1$. We shall show that the situation for (1.2) is significantly different.

We start in Section 2 by examining stationary solutions of (1.2). We give a definition of "coarsening" in Section 3, examine the special case of $(1.2)$ with $J(\cdot) \equiv 1$ to gain some insight into the behaviour in the more general case $J(\cdot) \geq 0$, and prove our main result (Theorem 5). We outline a numerical approximation method in Section 4 and show tests in both one and two space dimensions which back up and illustrate the main result. We finish with conclusions and observations.

\section{Stationary Solutions}

Consider the kinetic equation obtained by setting $\varepsilon$ to zero in the IDE (1.2) or the Allen-Cahn PDE (1.3),

$$
u_{t}=-f(u), x \in \Omega \text {. }
$$

The major difference between (1.3) and (1.2) is that the $O(\varepsilon)$ term in (1.3) is a singular perturbation of the kinetic equation, while in (1.2) it is a regular perturbation, since this term is a bounded linear operator.

It is not hard to show that (1.2) generates a dynamical system (a fact noted in [7]; (1.3) generates a semi-dynamical system) in various function spaces. We shall work in $L^{\infty}(\Omega)$; more precisely, the solutions $u(t)$ of (1.2) satisfy $u(t) \in$ $C^{1}\left([0, \infty), L^{\infty}(\Omega)\right)$. We shall denote the solution through an initial condition $\phi$ by $u(t, \phi)$.

There are two keys to our main theorem, Theorem 5. One is the following comparison principle stated in [7] (under more restrictive regularity conditions, but the $L^{\infty}$ statement goes through without any changes):

Lemma 1 ([7]) If $\phi, \psi \in L^{\infty}(\Omega)$ satisfy $\phi \geq \psi$ a.e., then $u(t, \phi) \geq u(t, \psi)$ a.e. for all $t \in[0, \infty)$. 
The other is the implicit function theorem which we examine below, following the treatment of [12] and noting that the stationary solutions are solutions of the equation $G(u, \varepsilon)=0$, where

$$
G(u, \varepsilon)=-f(u)+\varepsilon \int_{\Omega} J(|x-y|)(u(y)-u(x)) d x,
$$

a $C^{1}$ operator on $L^{\infty}(\Omega)$.

\section{Solutions for $\varepsilon=0$}

For $\varepsilon=0$, we see that there are three types of stationary solutions. Each stationary solution $u(x)$ can be written in the form

$$
u(x)=1 \chi_{A}(x)+(-1) \chi_{B}(x)+0 \chi_{\Omega \backslash(A \cup B)}(x),
$$

where $A, B$ are any measurable subsets of $\Omega$. If all the three sets in (2.2) have non-zero measure, we say that $u$ is a three-phase solution; if exactly one of them has zero measure, we say that $u$ is a two-phase solution; otherwise $u$ is one of the three one-phase solutions, \pm 1 or 0 .

We shall be in particularly interested in two-phase solutions on domains of size $|\Omega|=1$. For a fixed number $s \in(0,1)$, we shall use the following notation: by $(a, b)$, where $a, b$ can take the values $\pm 1,0$, we mean the set of stationary solutions $u$ that take the value $a$ on a set of measure $s$, and $b$ on a set of measure $1-s$. Thus $(1,0)$ and $(0,1)$ are the same sets if $s=1 / 2$ but different otherwise.

\section{Solutions for $\varepsilon>0$}

Theorem 2 Let $s \in(0,1)$ and let $u_{0}$ be an $(a, b)$ solution of $G(u, 0)=0$. There exists $\varepsilon^{*}>0$ such that every such solution has a locally unique continuation for all $0<\varepsilon<\varepsilon^{*}$.

Proof. Let us define

$$
\Omega_{+}\left(u_{0}\right)=\left\{x \in \Omega \mid u_{0}(x)>0\right\} ; \Omega_{-}\left(u_{0}\right)=\left\{x \in \Omega \mid u_{0}(x)<0\right\} ;
$$

and

$$
\Omega_{0}\left(u_{0}\right)=\left\{x \in \Omega \mid u_{0}(x)=0\right\} .
$$

In $L^{\infty}(\Omega)$ every $(a, b)$ solution $u_{0}$ of $G(u, 0)=0$ is isolated and hence locally unique. Since $-f^{\prime}\left(u_{0}\right) \in\{1,-2\}, D G^{-1}\left(u_{0}, 0\right)$ is the operator of multiplication by the $L^{\infty}$ function $g(x)=\chi_{\Omega_{0}\left(u_{0}\right)}(x)-1 / 2 \chi_{\Omega_{-}\left(u_{0}\right) \cup \Omega_{+}\left(u_{0}\right)}(x)$. Hence $\left\|D G^{-1}\left(u_{0}, 0\right)\right\| \leq 1$ for all $s \in(0,1)$ and by the implicit function theorem there is 
$\varepsilon^{*}>0$ such that $G(u, \varepsilon)=0$ has a locally unique solution $u(\varepsilon), u(0)=u_{0}$ for all $s$ in $(0,1)$ for all $0<\varepsilon<\varepsilon^{*}$.

Note that, as [12] remark, $\varepsilon^{*}$ is a common lower bound for the range of parameters for which $(a, b)$ solutions can be continued; the upper bounds are clearly dependent on $s$; a particular case is discussed in Section 3. Obviously, the above result holds for all $|\varepsilon|<\varepsilon^{*}$.

Since $G(u, \varepsilon)$ depends analytically on $\varepsilon$, regular perturbation expansions in $\varepsilon$ for solutions of $G(u, \varepsilon)$ converge for $\varepsilon$ sufficiently small. In Theorem 5 we shall need some information on qualitative properties of two-phase solutions for $\varepsilon$ small. This information is contained in the following lemma:

Lemma 3 If $u(0)=u_{0}$ is any $(1,0)$ solution, then for sufficiently small $\varepsilon>0$ we have that $u(\varepsilon)$ satisfies $u(\varepsilon)(x)=1-\varepsilon C_{1}(x)+O\left(\varepsilon^{2}\right)$ on $\Omega_{+}\left(u_{0}\right)$ and $u(\varepsilon)(x)=$ $-\varepsilon C_{2}(x)+O\left(\varepsilon^{2}\right)$ on $\Omega_{0}\left(u_{0}\right)$, where $C_{1}(x), C_{2}(x)$ are both $O(1)$ in $\Omega$ and positive.

Obviously, similar statements can be made for continuations of $(-1,0)$ solutions. The proof follows from the representation

$$
u(\varepsilon)(x)=u_{0}(x)-\varepsilon\left(\frac{\int_{\Omega} J(|x-y|)\left(u_{0}(y)-u_{0}(x)\right) d y}{1-3 u_{0}^{2}(x)}\right)+O\left(\varepsilon^{2}\right)
$$

and the non-negativity of $J(\cdot)$.

These results allow us to formulate a lemma that shows that there is a significant difference between (1.3), which has a compact attractor (see e.g. [9]), and (1.2), which does not.

Lemma 4 For sufficiently small $\varepsilon$ the attractor of the dynamical system generated by $(1.2)$ in $L^{\infty}(\Omega)$ is not compact.

Proof. Take a sequence $\left\{u_{0 n}\right\}, n=1, \ldots, \infty$ of solutions of the equation $G(u, 0)=$ 0 , such that $\left\|u_{0 i}-u_{0 j}\right\|_{\infty}=1$ if $i \neq j$. For $\varepsilon$ sufficiently small, let $u_{k}$ be the unique continuation of $u_{0 k}$. Then using Lemma 3 and the triangle inequiality, we have that for any two elements of the sequence, $u_{i}$ and $u_{j}$,

$$
\left\|u_{i}-u_{j}\right\|_{\infty} \geq 1-C \varepsilon
$$

for some constant $C$, so that the sequence $\left\{u_{n}\right\}, n=1, \ldots, \infty$ is not Cauchy. Hence the set of equilibria is not compact and therefore the attractor cannot be compact either.

An interesting question is whether there is a function-theoretic setting in which the attractor is compact. 


\section{Coarsening}

In this section we define what we mean by "coarsening", examine the coarsening of solutions of (1.2) in the case $J(\cdot) \equiv 1$ and then give a general result for the case $J(\cdot) \geq 0$. We start with the following definition.

Definition. Let $\phi \in L^{\infty}(\Omega)$, and let $u(t, \phi)$ be the solution through the initial condition $\phi(x)$ and assume that $\phi(x)$ changes sign in $\Omega$, so that both $\Omega_{+}(\phi)$ and $\Omega_{-}(\phi)$ have nonzero measure. We say that the solution does not coarsen if for each component $A_{+}$of the set $\Omega_{+}(\phi)$ and component $A_{-}$of $\Omega_{-}(\phi)$ there exist nonempty sets $B_{+}^{t}$ and $B_{-}^{t}$ such that $u(t, \phi)$ is positive on $B_{+}^{t}$ and negative on $B_{-}^{t}$ and $A_{+} \cap B_{+}^{t} \neq \emptyset$ and $A_{-} \cap B_{-}^{t} \neq \emptyset$ for all $t \in[0, \infty)$.

\section{The case $J(\cdot) \equiv 1$}

In this case one can have a relatively complete picture of the coarsening of solutions. First of all, the spectrum of the linearization around the stationary solution $u \equiv 0$ is easily computed. Linearizing, we have the eigenvalue problem

$$
\varepsilon \int_{\Omega} \phi d x=(\lambda-1+\varepsilon) \phi,
$$

so that at $\varepsilon=1$ we have a zero eigenvalue for which any function $\phi$ of zero mass is an eigenfunction.

Furthermore, one can draw the global bifurcation diagram for two-phase and three-phase solutions. Since we only require an understanding of two-phase solutions, we do not treat the other case here.

Clearly, all multi-phase solutions in this case are piecewise constant. Thus, any two-phase solution has the form

$$
u(x)=u_{1} \chi_{A}+u_{2} \chi_{\Omega \backslash A},
$$

where $A \subset \Omega$ and $u_{1} \neq u_{2}$. Setting $|A|=s, s \in(0,1)$, we obtain the following system of equations for $u_{1}, u_{2}$ :

$$
\left\{\begin{array}{l}
\varepsilon\left[s u_{1}+(1-s) u_{2}-u_{1}\right]-f\left(u_{1}\right)=0, \\
\varepsilon\left[s u_{1}+(1-s) u_{2}-u_{2}\right]-f\left(u_{2}\right)=0,
\end{array}\right.
$$

which is easily analysed using MAPLE. Eliminating $u_{1}$ we have:

$$
p\left(u_{2}, \varepsilon, s\right)=u_{2}^{6}+(3 \varepsilon s-2) u_{2}^{4}+\left(3 \varepsilon^{2} s^{2}-3 \varepsilon s+1\right) u_{2}^{2}+\varepsilon^{3} s^{2}-\varepsilon^{2} s^{2}=0 .
$$

Fixing $s$ and treating $\varepsilon$ as a bifurcation parameter, we obtain the bifurcation diagrams shown in Figures 1 and 2. Note that if $s \neq 1 / 2$ the second pitchfork bifurcation is broken. This is shown in Figure 2. 

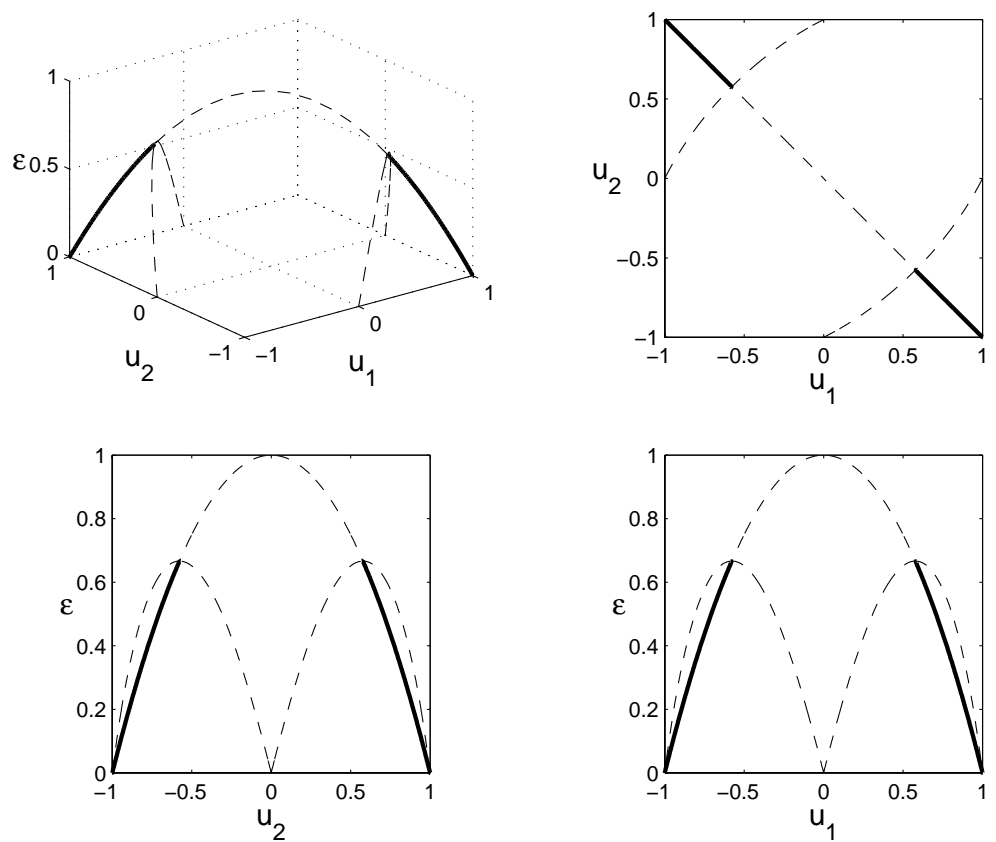

Figure 1: Solutions of (3.2) in the case $s=1 / 2, f(u)=u^{3}-u$. Broken lines denote unstable solutions and solid lines denote stable solutions.
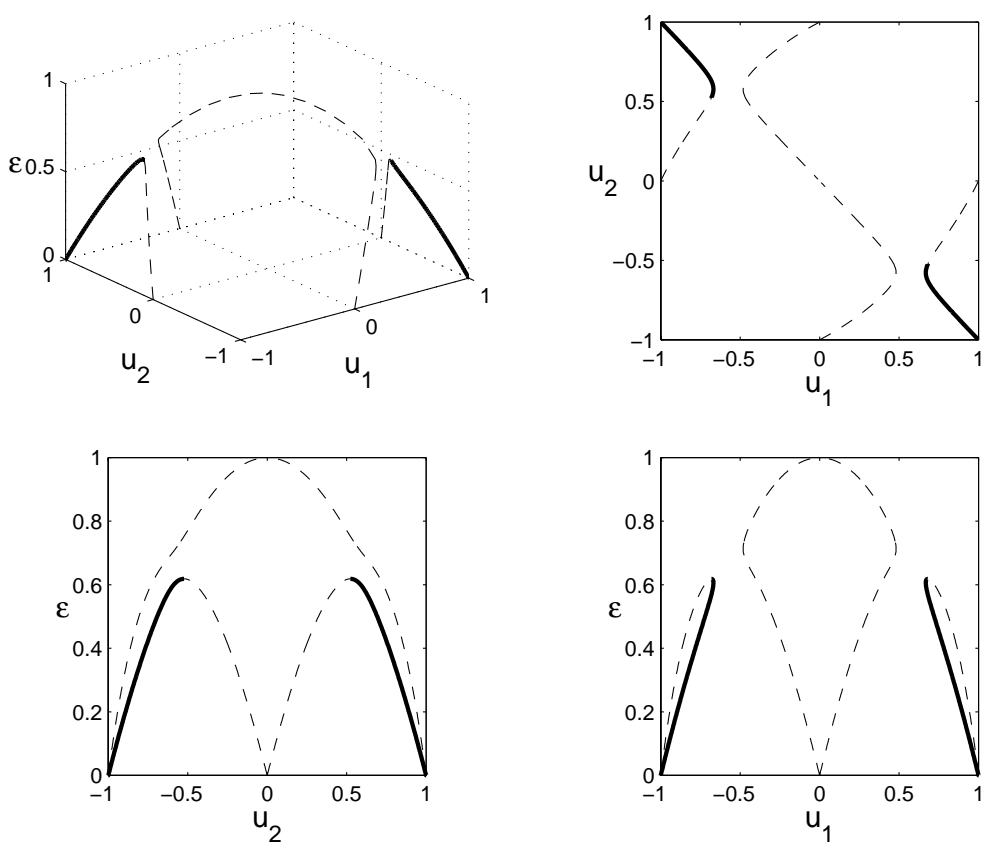

Figure 2: Solutions of (3.2) in the case $s=0.51, f(u)=u^{3}-u$. Broken lines denote unstable solutions and solid lines denote stable solutions. 
Obviously, the existence of solutions depends only on the value of $s$ and not on the geometry of the set $A$. It is also essential to realize that the branches of non-constant solutions above correspond to uncountable equivalence classes of stationary solutions. The solutions on the branches connecting to $u=\chi_{A}-\chi_{\Omega \backslash A}$ and $u=-\chi_{A}+\chi_{\Omega \backslash A}$ as $\varepsilon \rightarrow 0_{+}$are linearly stable in the $L^{\infty}(\Omega)$ norm. Then the heuristic explanation of disappearance of coarsening is that as we decrease $\varepsilon$, an initial condition that changes sign moves from being in the domain of attraction of a constant solution to that of one of the myriad non-constant ones, which for small enough $\varepsilon$ has the same pattern of change of sign as the initial condition itself. This is made clearer below.

Let us denote by $\varepsilon^{*}(s)$ the value of $\varepsilon$ at which a second bifurcation phenomenon (be it a pitchfork or a saddle-node bifurcation) occurs. We would like to know how $\varepsilon^{*}(s)$ depends on $s$, and, in particular, whether $\varepsilon^{*}(s) \rightarrow 0$ as $s \rightarrow 0$ or $s \rightarrow 1$. Computing the discriminant of (3.3) with respect to $u_{2}$, we see that it vanishes if $s$ and $\varepsilon$ satisfy the following relation:

$$
27 \varepsilon^{4} s^{2}(1-s)^{2}-18 \varepsilon^{2} s(1-s)+4 \varepsilon-1=0 .
$$

Thus this relation implicitly defines $\varepsilon^{*}(s)$, which is graphed in Figure 3. Clearly $\varepsilon^{*}(s) \nrightarrow 0$.

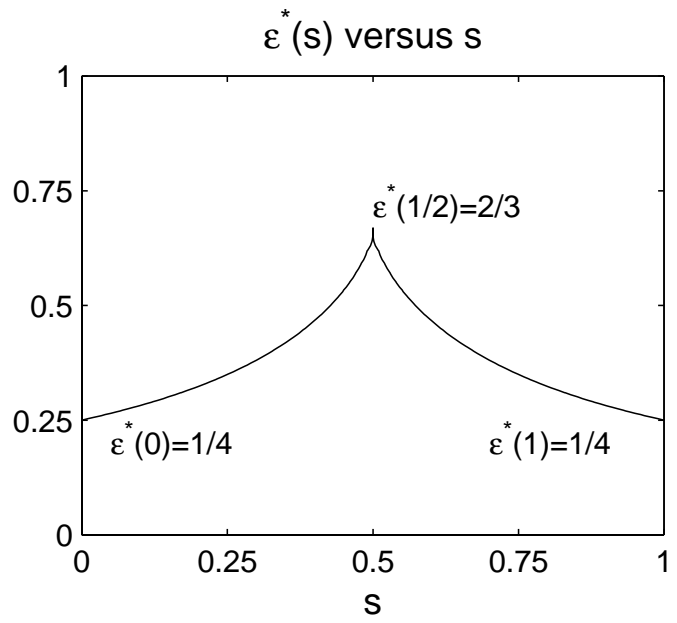

Figure 3: The relationship between $\varepsilon^{*}$ and $s$, the fraction of the space domain occupied by one phase of a two phase solution.

\section{The general case $J(\cdot) \geq 0$}

Now we state and prove our main theorem concerning coarsening. 
Theorem 5 For every kernel $J(\cdot) \geq 0$ and every initial condition

$\phi(x) \in L^{\infty}(\Omega)$ that changes sign, $\|\phi\|_{\infty}<1$, such that the measure of $\Omega_{0}(\phi)$ is 0 , there is a value $\varepsilon_{0}>0, \varepsilon_{0}=\varepsilon_{0}(\phi)$, such that for all $\varepsilon<\varepsilon_{0}$ the solution through $\phi(x)$ does not coarsen.

Proof. We start by making a number of definitions. Given an initial condition $\phi$ that changes sign, let $A_{+}^{i}$ be the $i$-th component (the number of which may be infinite) of $\Omega_{+}(\phi)$ and, similarly, let $A_{-}^{j}$ be the $j$-th component of $\Omega_{-}(\phi)$. We say that a pair $\left(u_{+}, u_{-}\right)$of stationary solutions of $(1.2)$ is a blocking pair for $\phi$ if

1. $u_{-} \leq \phi \leq u_{+}$, and

2. for each $i, j, u_{+}$is positive on a set that contains $A_{+}^{i}$ and negative on a subset of $A_{-}^{j}$; similarly, $u_{-}$is positive on a subset of $A_{+}^{i}$ and negative on set containing $A_{-}^{j}$.

Clearly, if a blocking pair exists for an initial condition $\phi$, then $u(t, \phi)$ cannot coarsen by Lemma 1 . Hence it only remains to show that for $\varepsilon$ sufficiently small every initial condition satisfying the assumptions of the theorem admits a blocking pair. However, this follows immediately from Theorem 2 and Lemma 3. See Figure 4 for an illustration of the construction.

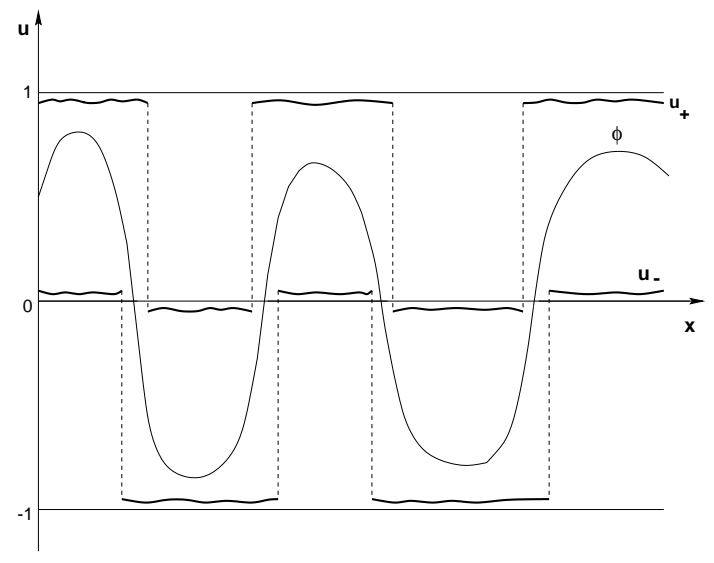

Figure 4: A blocking pair.

A weaker "partial non-coarsening" theorem can be proved in the case when $\left|\Omega_{0}(\phi)\right| \neq 0$; we leave the details to the reader. It should be possible to remove the assumption $\|\phi\|_{\infty}<1$ as well. 


\section{Numerical Approximation and Experiments}

\section{Numerical Approximation}

To illustrate how the numerical approximation of (1.2) is carried out, we consider the following example in one space dimension. Extension to more than one space dimension can be done in a similar way. The equation

$$
u_{t}=\varepsilon\left(\int_{0}^{1} J(|x-y|) u(y, t) d y-u(x, t) \int_{0}^{1} J(|x-y|) d y\right)-f(u)
$$

is approximated by partitioning the interval $[0,1]$ into a uniform subdivision of $N$ elements with node points $x_{j}=j \Delta x$ and mesh size $\Delta x=1 / N$. Then, bearing in mind that the solution need not be continuous, it is approximated as piecewise constant in space:

$$
u(x, t) \approx u_{j}(t) \text { for } x \in\left(x_{j-1}, x_{j}\right) .
$$

The approximate solution is substituted for the exact solution in (4.1) with $x=$ $x_{j-1 / 2}$ for $j=1, \ldots, N$, to obtain

$$
\frac{d u_{j}}{d t}=\varepsilon\left(\sum_{k=1}^{N} \bar{J}_{j, k} u_{k}-u_{j} \sum_{k=1}^{N} \bar{J}_{j, k}\right)-f\left(u_{j}\right)
$$

where

$$
\bar{J}_{j, k}=\Delta x J\left(\left|x_{j-1 / 2}-x_{k-1 / 2}\right|\right)=\Delta x J\left(\left|x_{j-k}\right|\right) \approx \int_{x_{k-1}}^{x_{k}} J\left(\left|x_{j-1 / 2}-y\right|\right) d y
$$

using the midpoint rule for approximate integration.

The approximation above can then be written as

$$
\underline{\dot{u}}=\varepsilon \Delta x A \underline{u}-\underline{f}(\underline{u})
$$

where the $j$ th component of $\underline{f}(\underline{u})$ is $f\left(u_{j}\right)$ and the matrix $A$ is symmetric with elements

$$
a_{j, k}= \begin{cases}J\left(\left|x_{j-k}\right|\right) & , j \neq k \\ J(0)-\sum_{r=1}^{N} J\left(\left|x_{j-r}\right|\right) & , j=k\end{cases}
$$

so that $A$ has real eigenvalues $\lambda_{j}$ (it is real and symmetric) with $\lambda_{j} \leq 0$ by the Gerschgorin circle theorem (since the row sums are zero). This ODE system is solved using a standard ODE package in MATLAB.

As an alternative approach to act as a check, the piecewise constant approximation of $u(x, t)$ in $x$ was replaced by approximation in terms of Chebyshev basis functions and using the pseudo-spectral method. The end result is an ODE system with similar structure producing almost identical results. 


\section{Numerical Experiments}

Figures 5 and 6 show results for the problem (1.2) in one space dimension. In these examples, the solution $u(x, t)$ is 1 -periodic in $x$ and starts from the initial data

$$
u(x, 0)=\sin \left(10 x^{2} \pi\right), \quad x \in[0,1] .
$$

The kernel function is the Gaussian

$$
J(x)=\sqrt{\frac{100}{\pi}} \exp \left(-100 x^{2}\right) .
$$

In Figure 5 we see the initial data (4.2) and final equilibrium solutions for a range of values of parameter $\varepsilon$. We see of course that when $\varepsilon=0$ the equilibrium is $u=\operatorname{signum}(u(x, 0))$, and when $\varepsilon$ increases the equilibrium solutions become simpler. When $\varepsilon$ is big enough the equilibrium solution is (in this example) the spatially homogeneous solution $u=+1$. Figure 6 shows the time evolution of the initial data (4.2) through to equilibrium for a fixed value of $\varepsilon$. The initial separation of the solution into domains where $u \approx \pm 1$ is followed by the collapse of the smaller scale domains.

Figure (7) shows snapshots of the time evolution of a solution of the problem (1.2) in two space dimensions. The solution is 1-periodic in each space direction (so we only show the unit square) and the kernel is

$$
J(x)=\frac{100}{\pi} \exp \left(-100|x|^{2}\right) .
$$

The initial value of $u$ in each subdivision of the unit square used in the numerical approximation is a randomly generated number between -1 and +1 . The figure shows the solution coarsening to form domains where $u \approx \pm 1$ and finally settling into a spatially inhomogeneous stable equilibrium.

\section{$5 \quad$ Remarks}

1. The assumption $J(\cdot) \geq 0$ was crucial in the comparison principle 1 and Lemma

3. Stationary solutions and their linearized stability in the case of $J(\cdot)$ changing sign are studied in [2].

2. We did not prove a stabilisation theorem. While it is obvious that $u_{t}>0$ at $t=0$ implies $u_{t}>0$ for all time, which would lead to a stabilisation result for a class of initial conditions, it is not clear how to prove a general stabilisation result for all $\phi \in L^{\infty}$. On one hand, there is not enough compactness in the system even to decide that $\omega$-limit sets (in strong topology) of initial conditions are non-empty, while on the other hand stabilisation in the kinetic equation is completely trivial. 
We hope that an approach via the Kuratowski measure of non-compactness [9] will resolve this issue.

3. Theorem 5 states that for small enough $\varepsilon$ the evolution system does not define a length-scale. In fact, the definition of non-coarsening can be taken to be a definition of spatial chaos. It is not clear what this implies for the suitability of equation (1.2) as a model of physical processes.

4. Finally, numerical experiments indicate that the equivalent of Theorem 5 is true also for the order parameter conserving version of (1.2). Clearly, methods based on comparison principle arguments do not work anymore and a fundamentally different approach is required in that case. 

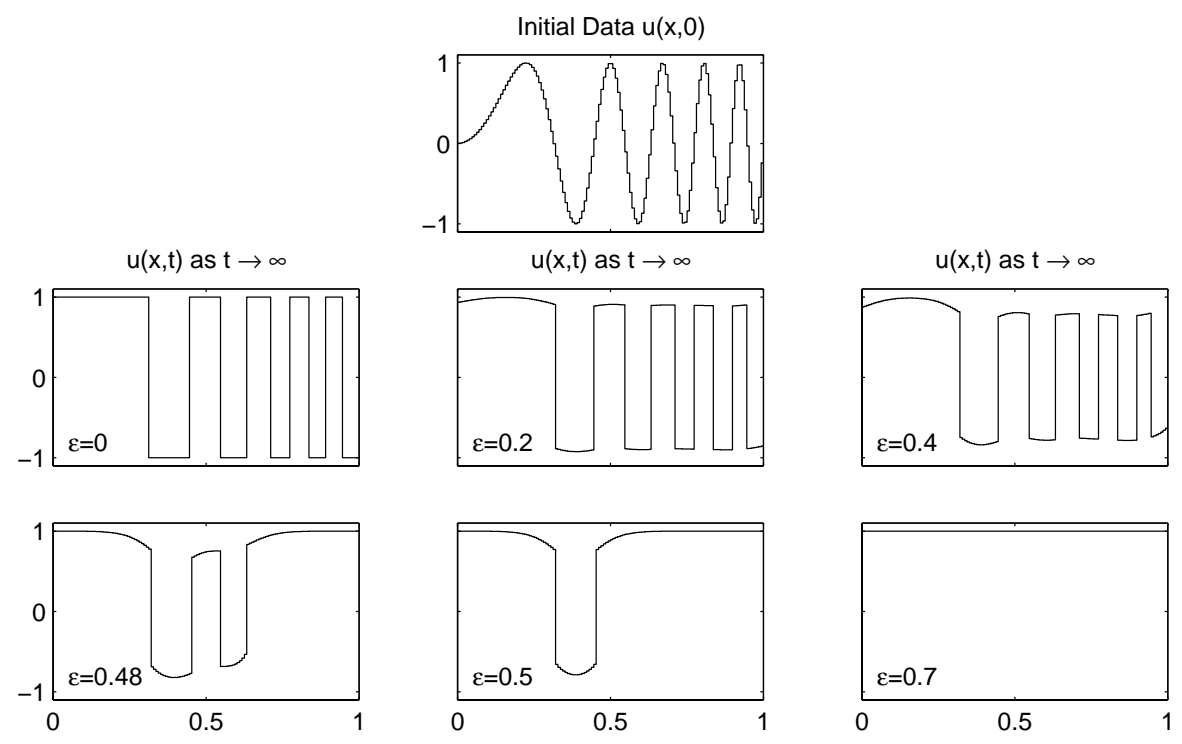

Figure 5: Equilibrium solutions of (1.2) for different values of $\varepsilon$ in the case $f(u)=u^{3}-u$ on the domain $\Omega=\mathbb{R}$ with kernel (4.3) and initial data (4.2). The solution is 1-periodic in $x .128$ elements are used in the numerical approximation.
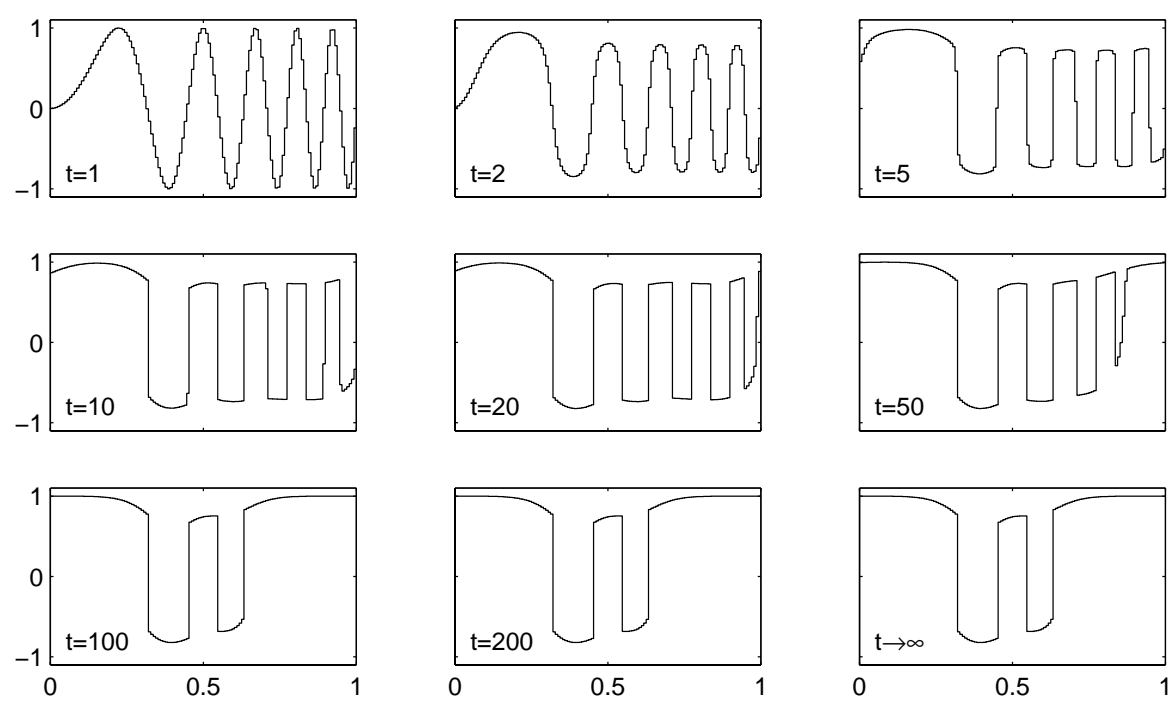

Figure 6: Time evolution of the solution of (1.2) with $\varepsilon=0.48$. All other details are as in the previous figure. 
$t=2$

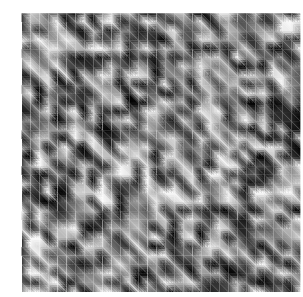

$\mathrm{t}=32$

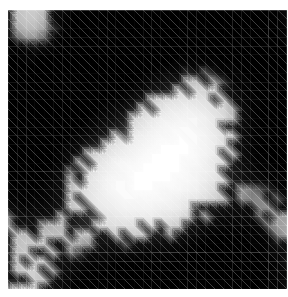

$\mathrm{t}=4$

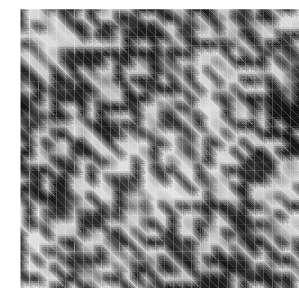

$\mathrm{t}=64$

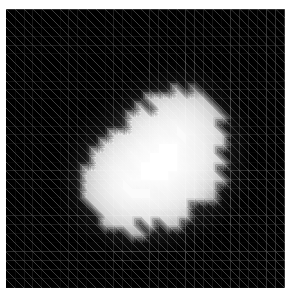

$t=8$

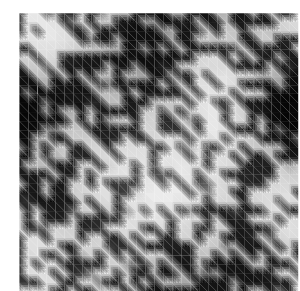

$\mathrm{t}=128$

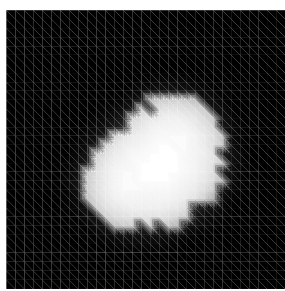

$t=16$

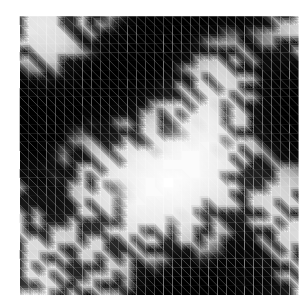

$t \rightarrow \infty$

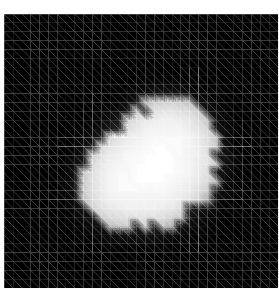

Figure 7: Time evolution of a solution of (1.2) in two space dimensions with $\varepsilon=0.55$, $f(u)=u^{3}-u$, kernel given by (4.4) and domain $\Omega=\mathbb{R}^{2}$. The solution is 1 -periodic in both space directions. The grid size for the numerical approximation is $32 \times 32$ and the initial data are randomly generated values between $[-1,1]$ on the grid. A grey scale is used to show values of $u$ between -1 (pure black) and +1 (pure white).

\section{References}

[1] P. W. Bates and A. Chmaj, A discrete convolution model for phase transitions, Arch. Ration. Mech. Anal. 150 (1999), 281-305.

[2] P. W. Bates and A. Chmaj, An integrodifferential model for phase transitions: stationary solutions in higher space dimensions, J. Stat. Phys. 95 (1999), 1119 1139.

[3] P. W. Bates, P. C. Fife, X. Ren, and X. Wang, Traveling waves in a convolution model for phase transitions, Arch. Ration. Mech. Anal. 138 (1997), 105-136.

[4] J. Carr and R. L. Pego, Metastable patterns in solutions of $u_{t}=\varepsilon^{2} u_{x x}-f(u)$, Comm. Pure Appl. Math. 42 (1989), 523-576.

[5] C. M. Elliott, A. R. Gardiner, I. Kostin, and Bainian Lu, Mathematical and numerical analysis of a mean-field equation for the Ising model with Glauber dynamics, in: Chaotic Numerics, ed. P. Kloeden and K. J. Palmer, AMS 1994.

[6] P. C. Fife, Models for phase separation and their mathematics, in Nonlinear Partial Differential Equations and Applications, M. Mimura and T. Nishida, eds., Kinokuniya Pubs., Tokyo, in press. 
[7] P. C. Fife, An integro-differential analog of semilinear parabolic PDEs, in: Partial Differential Equations and Applications, pp. 137-145, Marcel Dekker, 1996.

[8] G. Fusco and J. K. Hale, Slow motion manifolds, dormant instability and singular perturbations, Dynamics Diff. Equations 1 (1989), 75-94.

[9] J. K. Hale, Asymptotic Behaviour of Dissipative Systems, Math. Surveys and Monographs 25 A.M.S. 1988.

[10] J. M. Hyde, A. P. Sutton, J. R. G. Harris, A. Cerezo, and A. Gardiner, Modelling spinodal decomposition at the atomic scale: beyond the Cahn-Hilliard model, Modelling Simul. Mater. Sci. 4 (1996), 33-54.

[11] A. G. Khachaturyan, Theory of structural transformations in solids, Wiley, New York 1983.

[12] R. S. Mackay and J.-A. Sepulchre, Multistability in networks of weakly coupled bistable units, Physica 82D (1995), 243-254.

[13] O. Penrose, Statistical mechanics and the kinetics of phase separation, in: $M a-$ terial Instabilities in Continuum Mechanics and Related Mathematical Problems, J. M. Ball, ed., Clarendon Press, Oxford (1988), pp. 373-394.

[14] O. Penrose, A mean-field equation of motion for the dynamic Ising-model, J. Stat. Phys. 63 (1991), 975-986.

[15] J. D. van der Waals, The thermodynamic theory of capillarity flow under the hypothesis of a continuous variation in density, Verhandelingen der Koninklijke Nederlandsche Akademie van Wetenschappen te Amsterdam 1 (1893), 1-56. 